\title{
Theoretical investigation of InAs/GaSb type-II pin superlattice infrared detector in the mid wavelength infrared range
}

U. Kaya, M. Hostut, A. Kilic, S. Sakiroglu, I. Sokmen, Y. Ergun, and A. Aydinli

Citation: Journal of Applied Physics 113, 083717 (2013);

View online: https://doi.org/10.1063/1.4793787

View Table of Contents: http://aip.scitation.org/toc/jap/113/8

Published by the American Institute of Physics

\section{Articles you may be interested in}

Electrical modeling of InAs/GaSb superlattice mid-wavelength infrared pin photodiode to analyze experimental dark current characteristics

Journal of Applied Physics 116, 113101 (2014); 10.1063/1.4895983

"N" structure for type-II superlattice photodetectors

Applied Physics Letters 101, 073505 (2012); 10.1063/1.4745841

Midinfrared absorption by InAs/GaSb type-II superlattices

Journal of Applied Physics 105, 013115 (2009); 10.1063/1.3058692

Insight into the split and asymmetry of charge distribution in biased M-structure superlattice

Applied Physics Letters 111, 053503 (2017); 10.1063/1.4996863

Band-gap corrected density functional theory calculations for InAs/GaSb type II superlattices

Journal of Applied Physics 116, 214301 (2014); 10.1063/1.4903063

Absorption properties of type-II InAs/InAsSb superlattices measured by spectroscopic ellipsometry

Applied Physics Letters 106, 061907 (2015); 10.1063/1.4908255

\section{Scilight}

Sharp, quick summaries illuminating the latest physics research

\section{Sign up for FREE!}

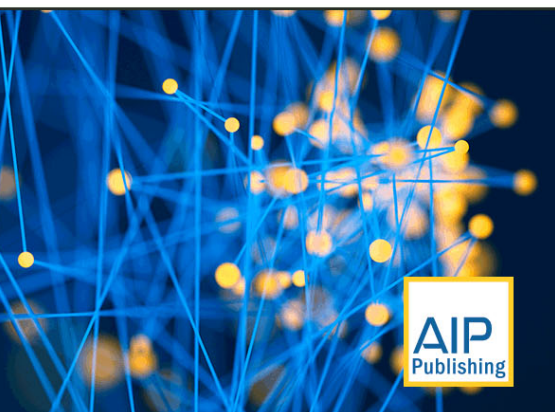




\title{
Theoretical investigation of InAs/GaSb type-II pin superlattice infrared detector in the mid wavelength infrared range
}

\author{
U. Kaya, ${ }^{1}$ M. Hostut, ${ }^{2}$ A. Kilic, ${ }^{3}$ S. Sakiroglu, ${ }^{4}$ I. Sokmen, ${ }^{4}$ Y. Ergun, ${ }^{3, a)}$ and A. Aydinli ${ }^{5}$ \\ ${ }^{1}$ Department of Electrical and Electronics Engineering, Anadolu University, \\ Iki Eylul Kampusu, TR-26555 Eskisehir, Turkey \\ ${ }^{2}$ Department of Secondary Education of Science \& Mathematics, Faculty of Education, \\ Akdeniz University, TR-07058 Antalya, Turkey \\ ${ }^{3}$ Department of Physics, Anadolu University, Yunusemre Kampusu, TR-26470 Eskisehir, Turkey \\ ${ }^{4}$ Department of Physics, Dokuz Eylul University, Kaynaklar Kampusu, TR-35160 Izmir, Turkey \\ ${ }^{5}$ Department of Physics, Bilkent University, TR-06800 Ankara, Turkey
}

(Received 4 December 2012; accepted 11 February 2013; published online 28 February 2013)

\begin{abstract}
In this study, we present the theoretical investigation of type-II InAs/GaSb superlattice p-i-n detector. Kronig-Penney and envelope function approximation is used to calculate band gap energy and superlattice minibands. Variational method is also used to calculate exciton binding energies. Our results show that carriers overlap increases at $\mathrm{GaSb} / \mathrm{InAs}$ interface on the higher energy side while it decreases at InAs/GaSb interface on the lower energy side with increasing reverse bias due to shifting the hole wavefunction toward to the GaSb/InAs interface decisively. Binding energies increase with increasing electric field due to overall overlap of electron and hole wave functions at the both interfaces in contrast with type I superlattices. This predicts that optical absorption is enhanced with increasing electric field. (C) 2013 American Institute of Physics. [http://dx.doi.org/10.1063/1.4793787]
\end{abstract}

\section{INTRODUCTION}

Type-II superlattice (T2SL) based on InAs/GaSb material system has been widely investigated as a potential candidate for advanced third generation infrared photon detector technology, since first proposed by Smith and Mailhiot. ${ }^{1} \mathrm{~T} 2 \mathrm{SL}$ material system enables special distributions of InAs and $\mathrm{GaSb}$ layers where conduction band of the InAs stays below the valence band of the GaSb. The overlap of electron wave functions in the InAs layers and the confinements of hole wave functions in the GaSb layers through the superlattice results in the formation of an electron and hole minibands in the conduction and valence bands, respectively. As a result, a spatially indirect bandgap opens, which is smaller than the band gap of the constituents. ${ }^{2}$ The effective band gap of the $\mathrm{SL}$ is defined as the energy separation between the lowest conduction and the highest valence minibands. The absorption wavelength can be adjusted from approximately $3-30 \mu \mathrm{m}$ by altering layer thickness to cover wavelength range from mid to very long infrared for infrared detection. ${ }^{3}$ InAs/GaSb T2SLs show significant development and prove viable alternatives to $\mathrm{HgCdTe}$ detectors and quantum well infrared detectors (QWIPs). They also show flexible designs of band structures which promise optical and electrical properties comparable to $\mathrm{HgCdTe}$ detectors with higher effective mass of electrons and holes, slower Auger recombination rate, lower dark current, and higher operating temperature. ${ }^{4}$ Other advantages are their lower thermal generation rate and normal incidence operation, in contrast with QWIPs in intersubband transition. All these advantages and technological requirements make them desirable in military, civilian applications in

\footnotetext{
a) Author to whom correspondence should be addressed. Electronic mail: yergun@anadolu.edu.tr.
}

night vision, reconnaissance, guidance, ranging, industrial thermography, medical imaging, meteorological research, and communication systems. There have been numbers of studies reported on focal plane array (FPA) applications for high quality T2SL structures up to date these includes single colour and dual colour InAs/GaSb SL in mid-wavelength and long wavelength infrared spectral range. .,3,5,6 $^{2}$

On the other hand, defining the spatial band alignment of InAs/GaSb T2SL as well as band gap energies and SL minibands are crucially important to obtain carrier wave functions and overlap integrals that effect the optical transition and carrier transport. Recently, we have investigated carriers overlap on InAs/AlSb/GaSb based T2LS p-i-n photodiode where $\mathrm{GaSb} / \mathrm{InAs}$ layers are confined by thin $\mathrm{AlSb}$ barriers. We found that the AlSb barriers shift the carriers in to $\mathrm{GaSb} / \mathrm{InAs}$ interfaces to perform strong overlap under reverse bias voltage. Our calculations of electron-hole wavefunction overlap integrals show that the absorption at the interface increases by about 25\% when an AlSb barrier is used Refs. 7 and 8. In this study, we perform band alignment of InAs/GaSb T2SL p-i-n structure with superlattice minibands and band-gap energies by applying Kronig-Penney ${ }^{9}$ and envelope function approximation (EFA) model as used in our previous works. ${ }^{7,8}$ Electron and hole wave functions in the junction region are obtained to investigate overlap integrals at both InAs/GaSb and GaSb/InAs interfaces under applied bias voltage. Beside this, we also investigate exciton binding energies to understand how e-hh coupling is affected by applied reverse bias at cryogenic temperatures. The paper is organized as follows. We outline the method to calculate energy band diagram of InAs/GaSb based T2SL p-i-n structure in Sec. II. Overlap wave functions and binding energy results are presented and discussed in Sec. III. Major conclusions are drawn for this study in Sec. IV. 


\section{THEORETICAL CONSIDERATION}

In this work, miniband structure of InAs/GaSb based type-II SL are calculated by Kronig-Penney model and EFA $^{9-11}$ given as without strain effect that the lattice mismatch, which is about $\Delta a / a=0.3-0.4 \%$, is acceptable for lattice periodicity. The EFA model is valid at the periodical boundary conditions. On the other hand, we have motivated by the recent work in which k.p theory carried out on InAs/ GaSb Type-II SL structure where electrons and holes are localised in InAs and GaSb layers, respectively. The overlap of electron wave functions between adjacent InAs layers results in the formation of electron minibands in the conduction band. Heavy holes are largely confined in the $\mathrm{GaSb}$ layers. The effective band gap of the SL is defined as the energy separation between the lowest conduction and the highest valence minibands. The SL wave functions behave like Kronig Penney plane waves out of depletion region. ${ }^{12,13}$ The suitable way to obtain pin structure and wave function spreading in the junction region is combination of EFA and Kronig Penney plane waves with this method, where real space electron and hole confinement in energy is seen under external applied voltage. Doping concentrations of n-InAs/ $\mathrm{GaSb}$ SL and p-InAs/GaSb SL are $\mathrm{Nd}=5 \times 10^{17} \mathrm{~cm}^{-3}$ and $\mathrm{Na}=1.5 \times 10^{17} \mathrm{~cm}^{-3}$, respectively. In the calculation energy zero is taken the conduction band edge of InAs. Fermi level for electrons in $n$-side is calculated by solving two dimensional electron density equations of $n_{2 D}=N_{d}\left(L_{w}+L_{b}\right)$ and $n_{2 D}^{i}=\frac{m_{e}^{*} k_{B} T}{\pi \hbar^{2}} \operatorname{In}\left[\operatorname{Exp}\left(E_{F}-E_{i}^{e}\right) / k_{B} T+1\right]$ together. Similarly, Fermi level for holes in p-side is also calculated by solving two dimensional hole density equations of $p_{2 D}=$ $N_{a} L_{b}(G a S b) \quad$ and $\quad p_{2 D}^{i}=\frac{m_{h}^{*} k_{B} T}{\pi \hbar^{2}} \operatorname{In}\left[\operatorname{Exp}\left(E_{i}^{h}-E_{F}\right) / k_{B} T+1\right]$. Where $\hbar$ is the Plank constant, $m^{*}$ is the effective mass of electron in ground state energy level of quantum well, $k_{B}$ is the Boltzmann constant, $T$ is the temperature in Kelvin, $E_{i}^{e}$ and $E_{i}^{h}$ are $i_{\text {th }}$ subband energy states below electron and hole subband band minima, respectively, $L_{w}$ is the well width and $L_{b}$ is the barrier width. For the given donor and acceptor densities, Fermi energy level of the pn junction takes place $7 \mathrm{meV}$ below the electron subband band minima in $\mathrm{n}$ side and $87 \mathrm{meV}$ above the hole subband maxima in $\mathrm{p}$ side, respectively. To calculate of the band bending of $p-n$ junction, procedure of the method is as follows. Electrical potential of this region as shown in Figure 1 is given as

$$
V(z)=\left\{\begin{array}{ll}
0 & z \leq-z_{n} \\
-\frac{q N_{d}}{2 \varepsilon_{o} \varepsilon_{n}}\left(z+z_{n}\right)^{2} & -z_{n}<z<0 \\
-\frac{q N_{d}}{2 \varepsilon_{o} \varepsilon_{p}}\left(z^{2}-z z_{p}\right)-\frac{q N_{d}}{2 \varepsilon_{o} \varepsilon_{n}} z_{n}^{2} & 0<z<z_{p} \\
-V_{o} & z \geq z_{p}
\end{array},\right.
$$

where $\mathrm{z}_{n}$ ve $\mathrm{z}_{p}$ are defined as depletion widths of $\mathrm{n}$ - and p-sides, respectively, $q$ is absolute value of electron charge and $\varepsilon_{o}$ is dielectric permittivity for free space, and $\varepsilon_{o} \varepsilon_{p}\left(\varepsilon_{o} \varepsilon_{n}\right)$ is p-side (n-side) dielectric permittivity. As seen from Fig. 1, $z=-z_{n}$ is taken to be centre of coordinate $\left(z \rightarrow z-z_{n}\right.$ in

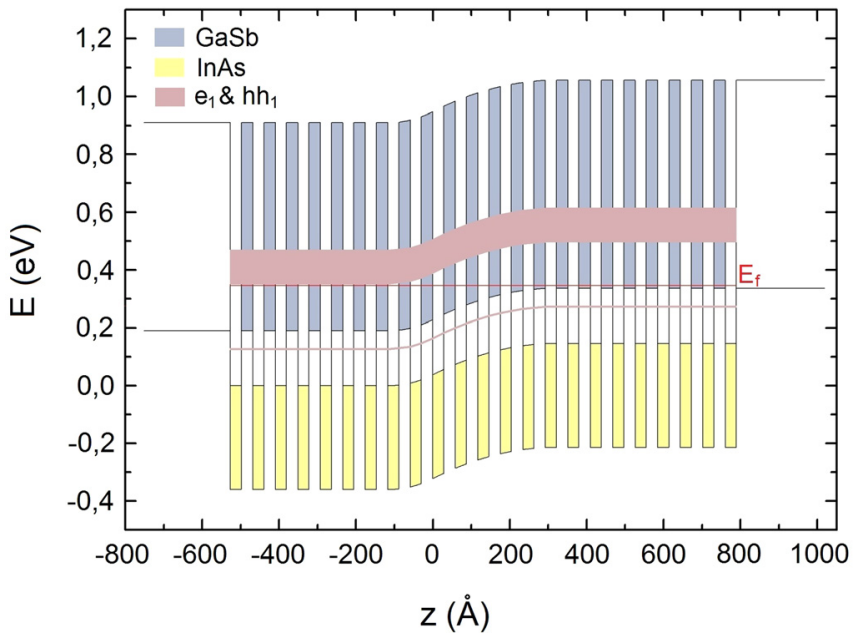

FIG. 1. Band alignment of InAs/GaSb SL pin junction with electron and hole minibands. SL consists of 22 periods of $8 \mathrm{ML}$ InAs/ $8 \mathrm{ML} \mathrm{GaSb}$.

Eq. (1)). $V_{o}$ is built-in potential of junction region which can be obtained by $\frac{\left(E_{g a p}^{e f f}-E_{f p}+E_{f n}\right)}{q}$. Where $E_{g a p}^{e f f}$ is the energy difference between electron and hh subband minima, $E_{f p}$ and $E_{f n}$ are Fermi levels in n-side of conduction and p-side of the valence band edges, respectively. The solution of pn junction is straight forward and can be find in any text books. By using potential and charge balance equations, electric field $(F)$ dependent second order of depletion width expression is given as

$$
\frac{q^{2} N_{a}}{2 \varepsilon_{o} \varepsilon_{p}}\left(\frac{N_{a}}{N_{d}}+1\right) z_{p}^{2}-e F z_{p}-E_{F n}+E_{F p}=0 .
$$

In the InAs/GaSb based T2SL system hole wave function in $\mathrm{GaSb}$ layer and electron wave function in InAs layer are confined in the same interface by revealing an effective excitonic behavior. The total Hamiltonien of the system in the $\mathrm{z}$-direction, which is growth direction, is given by

$$
H=H_{e z}+H_{e h}+\frac{P_{X}^{2}+P_{Y}^{2}}{2 M}+\frac{p_{x}^{2}+p_{y}^{2}}{2 \mu}-\frac{e^{2}}{\varepsilon r},
$$

where $H_{e z}$ and $H_{e h}$ electron(hole) Hamiltonian in z-direction while $\frac{P_{X}^{2}+P_{Y}^{2}}{2 M}$ is in $\perp$ plane kinetic energy operator of center of mass $\left(M=m_{e}+m_{h}\right)$ and $\frac{p_{x}^{2}+p_{y}^{2}}{2 \mu}$ is also kinetic energy operator for reduced effective mass $\left(\mu=m_{e} m_{h} /\left(m_{e}+m_{h}\right)\right)$ of electron and hole. Last term on the right hand side in Eq. (3) is excitonic Coulomb potential energy that will be computed. The total exciton wave functions of the system are normalized to ground state of hydrogen atom (s-states)

$$
\psi\left(\vec{r}_{e}, \vec{r}_{h}\right)=N \psi_{l e}\left(z_{e}\right) \psi_{l h}\left(z_{h}\right) \exp \left[-\frac{\rho}{\alpha}\right] .
$$

Therefore, the equation of eigenvalue are given

$$
H \psi\left(\vec{r}_{e}, \vec{r}_{h}\right)=E \psi\left(\vec{r}_{e}, \vec{r}_{h}\right) .
$$

In Eq. (4), where $\psi_{l e}\left(z_{e}\right)$ and $\psi_{l h}\left(z_{h}\right)$ are the confined electron and hole wave functions, respectively. $N$ is the normalization 
constant, $\rho$ and $\alpha$ are in plane coordinate $(x, y)$, respectively, and variational parameter which is a measure of exciton radius. The total exciton energy of $E_{1 S}\left(l_{e}, l_{h}\right)$ is calculated by variational method as follows: ${ }^{14}$

$$
E_{1 S}=\frac{\langle\psi|H| \psi\rangle}{\langle\psi \mid \psi\rangle} .
$$

Here the exciton energy of $E_{1 S}$ is given by

$$
E_{1 S}=E_{1 e}+E_{1 h}+E_{1 S}^{B} .
$$

The solution of Eqs. (3), (4), and (6) gives formally binding energy as

$$
E_{1 S}^{B}=\left\langle\psi\left|\frac{e^{2}}{\varepsilon \sqrt{\rho^{2}+\left(z_{e}-z_{h}\right)^{2}}}-\frac{p_{\perp}^{2}}{2 \mu}\right| \psi\right\rangle .
$$

By minimization of minimizational parameter of $\alpha$, exciton binding energy is obtained by

$$
\begin{aligned}
E_{1 S}^{B}= & \min _{\alpha}\left[\frac{4 e^{2}}{\varepsilon \alpha^{2}} \iiint\left|\psi_{e}\right|^{2}\left|\psi_{h}\right|^{2}\right. \\
& \left.\times \frac{\rho e^{-\frac{2 \rho}{\alpha}}}{\sqrt{\rho^{2}+\left(z_{e}-z_{h}\right)^{2}}} d z_{e} d z_{h} d \rho-\frac{\hbar^{2}}{2 \mu \alpha^{2}}\right],
\end{aligned}
$$

$\alpha$ indicates effective of electron-hole exciton radius which is calculated by around 170-180 $\AA$ consistent with literature. ${ }^{14}$ In calculation, $z_{e}$ and $z_{h}$ are scaled by effective Bohr radius which is $16.68 \AA$ and energy is scaled by effective Rydberg energy of $0.431 \mathrm{eV}$. Finally, the electron-hole overlap integral, $F(0)$, is given by

$$
F(0)=\left|\int_{-\infty}^{+\infty} d z \psi_{e}(z) \psi_{h}(z)\right|^{2} .
$$

Especially at the long wave application, to increase e-hh overlap near the interface, a few AlSb mono layers can be inserted in the middle of GaSb (InAs) in GaSb/InAs superlattice. Thus, electron-hole confinement can be increased and alpha coefficient can be decreased.

\section{RESULTS AND DISCUSSION}

Calculated potential energy band profile of type-II InAs/ GaSb SL p-n junction with $28.5 \AA$ GaSb and $30 \AA$ InAs layers are given in Figure 1. Band widths of electron and hole minibands are calculated as 112 and $0.2 \mathrm{meV}$ in energy, respectively. Effective gap (e1-hh1) in the structure is $276 \mathrm{meV}$ which corresponds to cut-off wavelength of $4.5 \mu \mathrm{m}$ at $77 \mathrm{~K}$ shown in Figure 1. Effective masses of electrons (holes) in InAs and $\mathrm{GaSb}$ are given $m_{e}^{*}(\operatorname{InAs})=0.023 m_{o}\left(m_{h}^{*}(\operatorname{InAs})\right.$ $\left.=0.41 m_{o}\right)$ and $m_{e}^{*}(G a S b)=0.041 m_{o}\left(m_{h}^{*}(G a S b)=0.4 m_{o}\right)$ respectively, where $m_{o}$ is the free electron mass. Figure 2 shows electron band energy separation, heavy hole energy, and band gap of $\mathrm{GaSb}$ as a function of GaSb thickness taken InAs layer thickness of $30 \AA$. When GaSb thickness is increased, the electron subband minimum energy is increased while electron

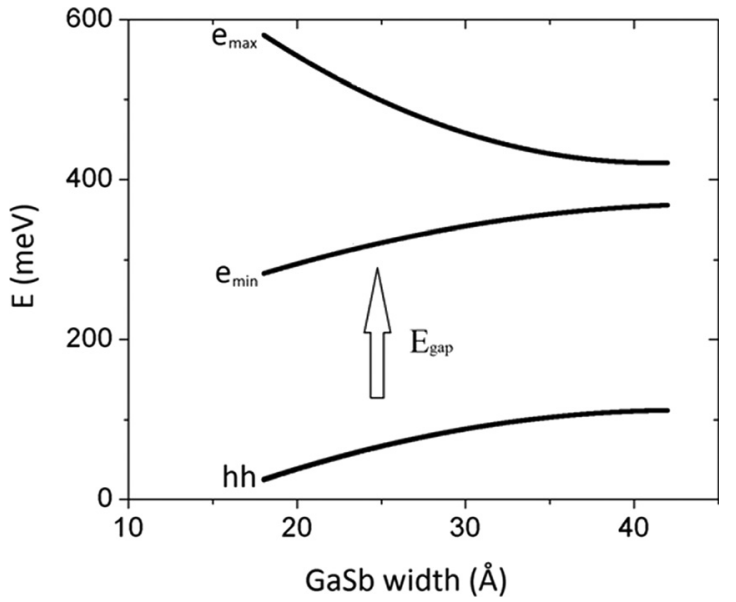

FIG. 2. Electron band seperation $\left(\mathrm{e}_{\max }-\mathrm{e}_{\min }\right)$ and heavy hole band energies of InAs/GaSb SL as a function of GaSb layer thickness for $30 \AA$ thickness of InAs layer.

subband maximum energy is decreased. That is the energy separation of electron band energy $\left(e_{\max }-e_{\min }\right)$, which is called band narrowing, is decreased with increasing GaSb thickness. The hh-band energy shown as a line is increased with increasing GaSb thickness. The gradients of the band curvatures of electron subband minimum and hh-band are almost same. Therefore, band gap energy is almost unchanged. Figure 3 shows the wave functions for two closest electron states and a heavy hole state in the junction region of InAs/GaSb T2SL p-i-n junction under reverse bias. The electron wave functions are mainly localized in adjacent InAs wells in $300 \AA$ range in the growth direction within the electron miniband. Heavy hole wave function is confined in the GaSb hole well within the $0.2 \mathrm{meV}$ hole miniband. Therefore, hole miniband width in energy is much smaller than that of electron miniband. In overlap calculations, we take the localized electron wave functions in adjacent InAs wells on the left $\psi_{e}^{j}(z)$ and right $\psi_{e}^{j+1}(z)$ hand side of the central GaSb hole well with confined hole wave function of $\psi_{h}^{j}(z)$ given in Figure 3. Overlap on the left and right hand side are defined as $\psi_{h}^{j}(z) \psi_{e}^{j}(z)$ and

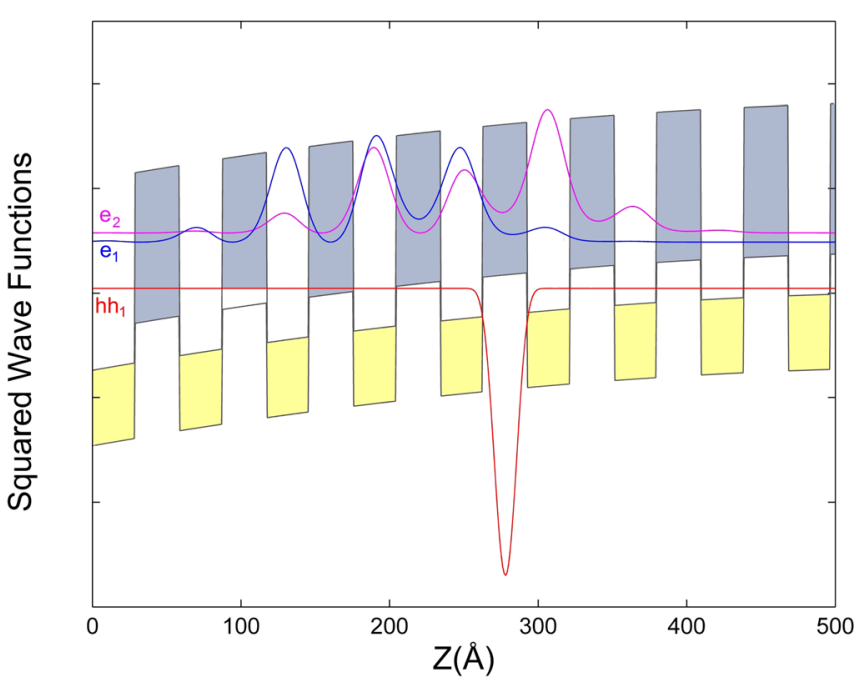

FIG. 3. Potential energy profile of the InAs/GaSb SL pin structure with electron and hole wave fuctions under reverse bias voltage. Carrier wave functions are given in energy as $\mathrm{e}_{1}=0.74 \mathrm{eV}, \mathrm{e}_{2}=0.78 \mathrm{eV}$, and $\mathrm{hh}_{1}=0.523 \mathrm{eV}$. 


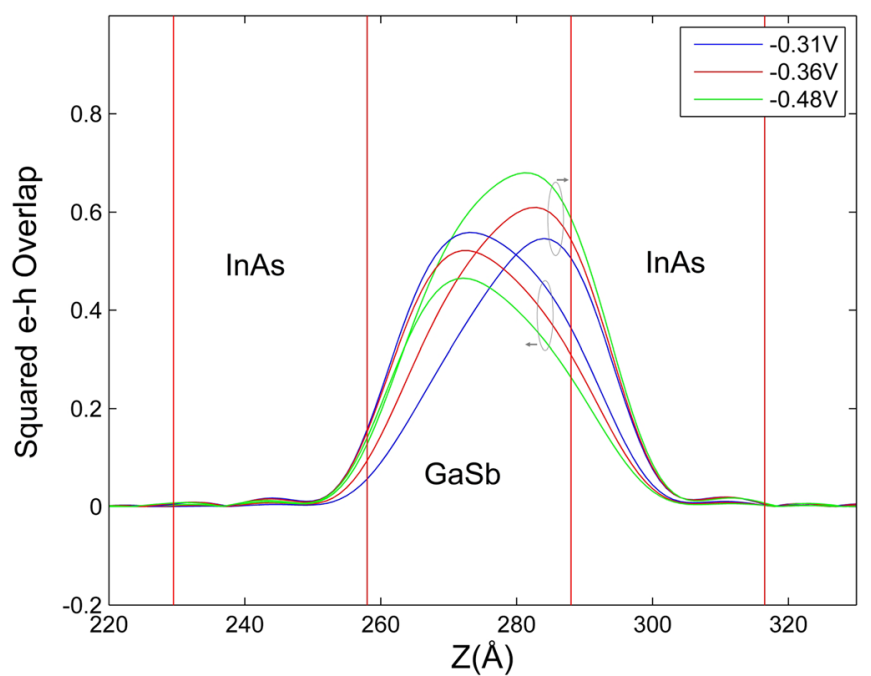

FIG. 4. Electron and hole overlaps from InAs/GaSb and GaSb/InAs interfaces under various applied bias voltages. The lower and the higher parts of curves indicate the overlaps at $\mathrm{InAs} / \mathrm{GaSb}$ and $\mathrm{GaSb} / \mathrm{InAs}$ interfaces, respectively.

$\psi_{h}^{j}(z) \psi_{e}^{j+1}(z)$, respectively. Where $j$ is periodicity number in the junction region. Figure 4 shows the squared electron-hole overlaps at the InAs/GaSb (on the left hand side) and GaSb/ InAs interfaces (on the right hand side) of the GaSb hole well at various bias voltages. We obtain that the overlap at the InAs/GaSb interface on the lower energy side is decreased while it is increased at the GaSb/InAs interface on the higher energy side of the junction under reverse bias. In fact, the hole wave function is pushed toward to the $\mathrm{GaSb} / \mathrm{InAs}$ interface by applied reverse bias to increase the overlap. Beside this, it has been observed that the overall overlap indicated as $\psi_{h}^{j}(z)\left(\psi_{e}^{j}(z)+\psi_{e}^{j+1}(z)\right)$ from both InAs/GaSb and GaSb/InSb interfaces are increased with increasing bias voltage. Figure 5 shows the two overall overlaps under potential energy of $0.8 \mathrm{meV}$ and $1.2 \mathrm{meV}$ per period. In the calculations of the overlaps, the hole wave functions are more decisive than the electron wave functions.

Spatially separation of electrons and holes from the type-II band alignment allows for the formation of excitons.

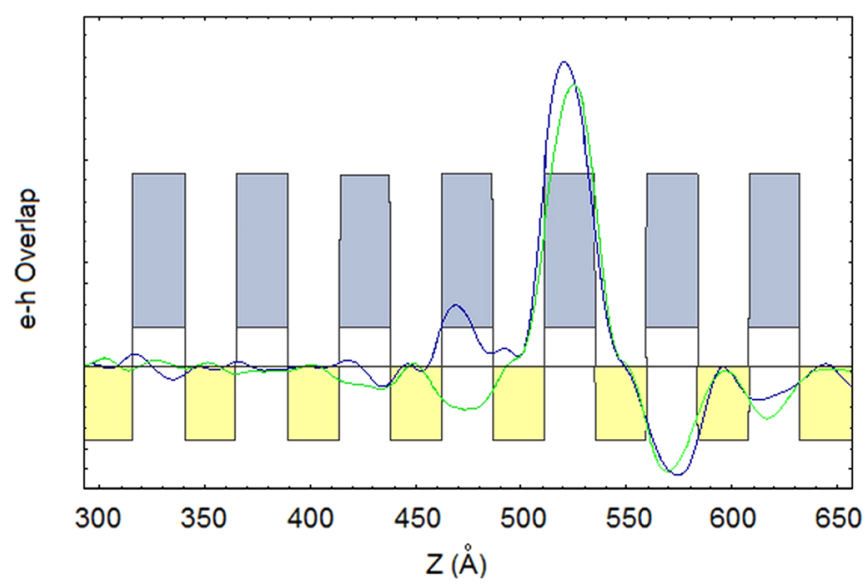

FIG. 5. The overall overlaps from InAs/GaSb and GaSb/InAs interfaces under the potential energies of 0.8 and $1.2 \mathrm{meV}$ per period given with green and blue colors, respectively.

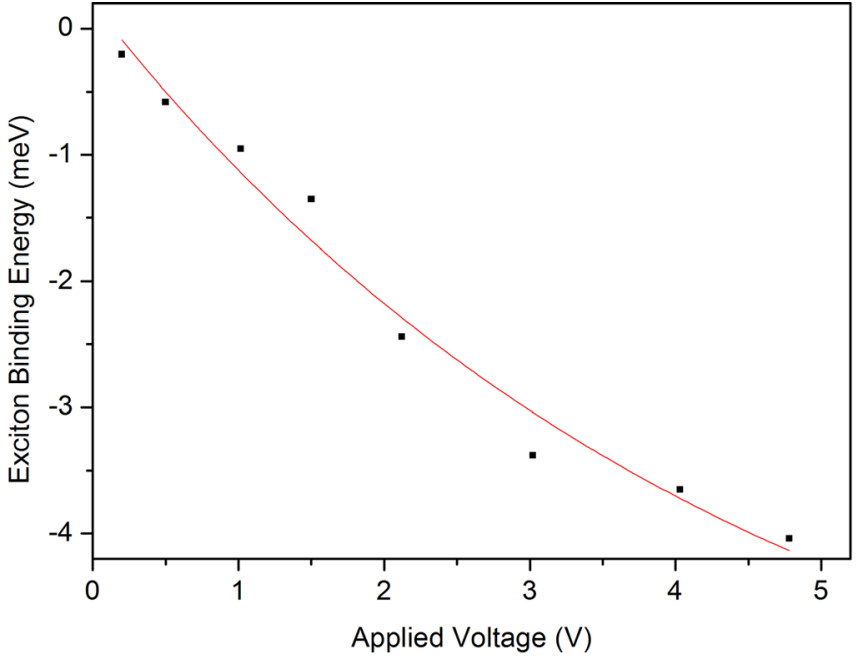

FIG. 6. Exciton binding energy as a function of applied voltage. Dots represent the obtained binding energies from calculations at given bias voltages.

Exciton binding energy depends on barrier/well widths and also type of structure for instance type-I and type-II. It is reported that exciton binding energy increases up to $5.62 \mathrm{meV}$ as the well width decreases from 70 to $10 \AA .{ }^{15} \mathrm{We}$ have plotted the exciton binding energy as a function of the applied voltage in Figure 6. As seen from the figure, exciton binding energy increases with increasing bias voltage since significant overlap is achieved at the interfaces with bias voltage. As a result, the exciton becomes confined at the $\mathrm{GaSb} / \mathrm{InAs}$ interfaces, leading to more binding. Superlattice with around $30 \AA$ well width, the exciton binding energy changes in the range of $0-4 \mathrm{meV}$ which is consistent with the results given in the literature. ${ }^{14,16}$ Obtained binding energies from the calculations ${ }^{14}$ given by dots are also shown in Figure 6. Exciton binding energy might be increased linearly with increasing applied bias voltages. The reason for the deviation is indicated as below

$$
V=V(S L)+V_{p n}\left(X_{n}(F)+X_{p}(F)\right)+e F z
$$

where the total potential $V$ contains superlattice potential $V(\mathrm{SL}), \mathrm{p}-\mathrm{n}$ junction potential $V_{p n}$ which changes as a function electric field dependence of $X_{n}$ and $X_{p}$, and additional potential relating with applied electric field. In the intrinsic region of the p-i-n SL structure the potential energy of InAs electron wells and GaSb hole wells (barriers) have a gradient not being in the order as the bias voltage is applied. Therefore, potential fluctuations cause the fluctuations from linearity for exciton binding energies as the applied bias voltage is increased. As a result, calculated values of exciton binding energies are deviated from the linearity.

\section{CONCLUSIONS}

The theoretical investigation of electronic miniband structure of $\mathrm{InAs} / \mathrm{GaSb}$ T2SL p-i-n diode is presented by employing Kronig-Penney model and EFA. We found that carriers overlap is increased at GaSb/InAs interface while it is decreased at the InAs/GaSb interface by increasing reverse bias. Exciton binding energy is also increased with 
increasing applied electric field. But it is very small (in the $0-4 \mathrm{meV}$ range) and unimportant especially in cryogenic temperatures for infrared applications. The results are promisingly motivate us to study $\operatorname{InAs} / \mathrm{Al}_{\mathrm{x}} \mathrm{Ga}_{1}{ }_{-\mathrm{x}} \mathrm{Sb} / \mathrm{GaSb}$ type-II p-i-n SL where strong overlap takes place at GaSb/InAs interface leading to higher absorption and detectivity as a future work.

\section{ACKNOWLEDGMENTS}

Y. Ergun acknowledges the support of TUBITAK and Anadolu University (Grants from Tubitak: 109T072 and BAP: 1104F073-1104F074, respectively). M. Hostut also acknowledges the support of Akdeniz University (BAP Grant: 2012.01.0110.002)

${ }^{1}$ D. L. Smith and C. Mailhiot, J. Appl. Phys. 62, 2545 (1987).

${ }^{2}$ M. Walther and G. Weimann, Phys. Status Solidi A 203, 3545 (2006).

${ }^{3}$ R. Rehm, M. Walther, J. Schmitz, F. Rutz, J. Fleißner, R. Scheibner, and J. Ziegler, Infrared Phys. Technol. 52, 344 (2009).
${ }^{4}$ J. B. Rodriguez, C. Cervera, and P. Christol, Appl. Phys. Lett. 97, 251113 (2010).

${ }^{5} \mathrm{M}$. Razeghi and B.-M. Nguyen, paper presented at the 6th International Workshop on Information Optics (WIO ‘07), (2007), Vol. 35.

${ }^{6}$ C. J. Hill, A. Soibel, S. A. Keo, J. M. Mumolo, D. Z. Ting, and S. D. Gunapala, Infrared Phys. Technol. 52, 348 (2009).

${ }^{7}$ O. Salihoglu, M. Hostut, T. Tansel, K. Kutluer, A. Kilinc, M. Alyoruk, C. Sevik, R. Turan, Y. Ergun, and A. Aydinli, Infrared Phys. Technol. (in press).

${ }^{8}$ O. Salihoglu, A. Muti, K. Kutluer, T. Tansel, R. Turan, Y. Ergun, and A. Aydinli, Appl. Phys. Lett. 101, 073505 (2012).

${ }^{9}$ L. L. Li, W. Xu, J. Zhang, and Y. L. Shi, J. Appl. Phys. 105, 013115 (2009).

${ }^{10}$ G. Bastard, E. E. Mendez, L. L. Chang, and L. Esaki, Phys. Rev. B 26, 1974 (1982)

${ }^{11}$ J. H. Roslund and T. G. Anderson, Superlattices Microstruct. 16, 77 (1994).

${ }^{12}$ F. Szmulowicz, H. Haugan, and G. J. Brown, Phys. Rev. B 69, 155321 (2004).

${ }^{13}$ R. Magri and A. Zunger, Phys. Rev. B 68, 155329 (2003).

${ }^{14}$ H. Sari, Y. Ergün, I. Sökmen, S. Elagöz, and N. Balkan, Semicond. Sci. Technol. 14, 412 (1999).

${ }^{15}$ A. J. Peter and C. W. Lee, Phys. Scr. 85, 015704 (2012).

${ }^{16}$ S. de-Leon and B. Laikhtman, Phys. Rev. B 61, 2874 (2000). 